\title{
Organización como proceso de la seguridad industrial e higiene ocupacional en la Industria petrolera
}

Organization as a process of industrial safety and occupational hygiene in the oil industry

\begin{tabular}{l|r}
\cline { 2 - 2 } & Boreidy Jiménez \\
\hline Artículo recibido en mayo 2020 & boreidyjimenez@gmail.com \\
Arbitrado en junio 2020 \\
Publicado en septiembre 2020 & ORCID: 0000-0003-4190-1027 \\
Industria Petrolera, Venezuela
\end{tabular}

RESUMEN

Palabras clave:

ABSTRACT

Keywords:
Toda organización debe mantener entre sus premisas para el éxito de sus negocios el cuidado, en lo que a gestión de seguridad industrial e higiene ocupacional se refiere. Desde esta perspectiva, el objetivo de esta investigación fue analizar el proceso de organización de la gestión de seguridad industrial e higiene ocupacional en la industria petrolera, según la percepción de los gerentes y líderes de las gerencias de Servicios Lacustre división Occidente. Metodológicamente se enmarcó en una investigación descriptiva, con diseño no experimental, de campo y transeccional. Se aplicó una encuesta mediante un cuestionario, el mismo quedó conformado 9 ítems con escala dicotómica, validado por el juicio de expertos, y de alta confiabilidad según el método de Küder - Richardson. Las frecuencias relativas se utilizaron para el análisis de los datos. Se concluye que estas gerencias otorgan moderada presencia al comité de seguridad industrial, responsabilidad y comunicación como indicadores del proceso.

Comité de seguridad industrial, comunicación, organización, responsabilidad, seguridad industrial e higiene ocupacional

Every organization must maintain among its premises for the success of its business, care, as far as industrial safety and occupational hygiene management is concerned. From this perspective, the objective of this research was to analyze the organization process of industrial safety and occupational hygiene management in the oil industry, according to the perception of the managers and leaders of the Lacustrine Services divisions West division. Methodologically it was framed in a descriptive investigation, with a non-experimental, field and transectional design. A survey was applied using a questionnaire, it was made up of 9 items with a dichotomous scale, validated by the judgment of experts, and highly reliable according to the Küder Richardson method. Relative frequencies were used for data analysis. It is concluded that these managements give a moderate presence to the industrial safety, responsibility and communication committee as indicators of the process.

Industrial safety committee, communication, organization, responsibility, industrial 


\section{INTRODUCCIÓN}

Hoy día, es importante la compatibilidad del ambiente laboral con las capacidades de cada trabajador, la prevención de los accidentes laborales, enfermedades ocupacionales, al fomento y mantenimiento de una cultura de bienestar físico, social, así como mental. De todo lo descrito anteriormente, se desprende el hecho de que en la actualidad muchas organizaciones buscan implantar sistemas de gestión en materia de seguridad industrial e higiene ocupacional que le permitan no sólo controlar y mitigar los riesgos laborales asociados a sus procesos y actividades, sino, además, cumplir con todo lo establecido en el marco legal de cada país, asegurarse de su conformidad de acuerdo a lo establecido en su política de seguridad y salud.

En este marco de ideas, la gestión de seguridad industrial e higiene ocupacional, según Aguilar (2011), es una ciencia dedicada al reconocimiento, evaluación y control de aquellos factores ambientales $\mathrm{o}$ tensiones emanados o provocados por el lugar de trabajo y que pueden ocasionar enfermedades, destruir la salud y el bienestar o crear algún malestar significativo entre los trabajadores o los ciudadanos de una comunidad.

Partiendo de lo anteriormente expuesto, se presenta esta investigación, en el contexto de la industria petrolera $y$ en específico en las gerencias de servicio lacustre, las cuales ha venido administrando el control de los riesgos en materia de seguridad, higiene, salud en el trabajo y ambiente a través del sistema de gerencia integral de riesgos (SIR); siendo aprobada esta para su implantación en abril del 2004, por la junta directiva de Petróleos de Venezuela S.A. (PDVSA, página oficial de internet 2019), a fin de establecer a través de ella un conjunto de requisitos interrelacionados para minimizar y controlar los riesgos relacionados a los procesos y actividades en materia de seguridad, higiene, salud en el trabajo y ambiente.

Estas gerencias, según un diagnóstico previo realizado por la investigadora, detecto una serie de debilidades en la gestión de seguridad industrial e higiene ocupacional de las gerencias de Servicios Lacustre división occidente, relacionadas a fallas en las inspecciones de las instalaciones por parte de un comité organizador, sin un responsable de garantizar el cumplimiento en materia de seguridad industrial así como de las leyes y normas que la regulan por parte del personal supervisorio, lo que acentúan la falta de cultura preventiva en cada uno de los trabajadores (as), quienes deben iniciar por comprender que la seguridad no depende de otros sino que es un valor de vida que cada uno debe internalizar y poner en práctica.

Partiendo de lo anteriormente expuesto, la investigadora abordada la presente investigación, a través de la siguiente pregunta: ¿Cómo es el proceso de organización en la gestión de seguridad industrial e higiene ocupacional presente en las gerencias de servicio lacustre de la industria petrolera división occidente?

\section{Organización como proceso de la seguridad industrial e higiene ocupacional}

Según la norma venezolana COVENIN 2260 (2004, p. 2), la seguridad industrial es

"Un conjunto de principios, leyes, criterios y normas formuladas, cuyo objeto fundamental es controlar el riesgo de accidentes que pudiesen 
derivar en lesiones a las personas, al igual que daños a los materiales y equipos intervinientes en el desarrollo de toda actividad productiva".

Mientras que en esta misma norma COVENIN 2260 (2004, P. 1), la higiene ocupacional se define como:

"La ciencia dedicada al conocimiento, evaluación y control de aquellos factores ambientales $\mathrm{O}$ tensiones emanadas $\mathrm{O}$ provocadas por o con motivo del trabajo pudiendo ser causantes de enfermedades, afectar la salud y el bienestar, o crear algún malestar significativo entre los trabajadores o los ciudadanos de la comunidad".

De igual manera, en opinión de Peralta (2010), la gestión de seguridad industrial e higiene ocupacional se ocupa de proteger la salud de los trabajadores, controlando el entorno del trabajo para reducir o eliminar riesgos, con respecto al ambiente toma en cuenta los aspectos físicos que rodea al trabajador mientras desempeña su cargo, en relación a la higiene ocupacional se encarga de proteger y promover la salud y el bienestar de los trabajadores, salvaguardando la comunidad así como el medio ambiente en general.

Asimismo, Hernández y otros (2005), consideran la higiene ocupacional como una herramienta importante dentro de las organizaciones ya que facilita la prevención de accidentes y enfermedades profesionales, coordinando para cada área de trabajo, las medidas preventivas dirigidas a minimizarlos riesgos de accidentes dentro del área laboral. Es importante resaltar que la seguridad industrial e higiene ocupacional, permite a la organización brindara los trabajadores cursos de adiestramiento en el uso de los materiales para evitar accidentes, charlas de prevención, guías o folletos donde se detallen los pasos para realizar las tareas programadas, controlar el cumplimiento de las normativas en seguridad.

A criterio de la investigadora, la de seguridad industrial e higiene ocupacional es un sistema que abarca los procedimientos para identificar, evaluar y controlar los agentes nocivos y factores de riesgo, presentes en el medio ambiente laboral, los cuales deberán incorporarse en las gerencias de servicio lacustre de la industria petrolera división occidente, a través de técnicas permanentes, sistemáticas, de mejora continua, bajo la aplicación de estrategias, en pro de la salud física, mental y emocional de los trabajadores en el ejercicio de su actividad laboral.

Ahora bien, toda empresa debe determinar y definir la manera en que, desde el punto de vista organizacional, funcionará distribuyendo las tareas y los puestos de trabajo para cada uno de los eslabones que la deben conformar.

Según Robbins y Ocultar (2005), definen la organización como la función de la administración que consiste en determinar que tareas hay que hacer, quien lo hace, como se agrupan, quien rinde cuentas a quién y donde se toman las decisiones. Para Cortés (2007), la organización es aquella que se encarga de promover la cooperación entre los diferentes departamentos definidos dentro de la empresa, garantizando la comunicación de la información a través de ella.

En otra perspectiva, Koontz y otros (2008), conceptualizan la organización como una estructura de funciones o puestos definidos y formalizados. En el contexto de la seguridad industrial e higiene ocupacional Hernández y otros (2003) refieren que se debe disponer de una organización y de los medios necesarios que aseguren el cumplimiento del deber de protección empresarial (conjunto de relaciones y 
personas que integradas en la estructura organizativa de la empresa asegura el cumplimiento de los objetivos preventivos).

Refieren los citados autores que las modalidades de organización vienen reguladas por la Ley de Prevención de Riesgos Laborales (1995), en ella se establece que la dirección de la empresa fijará la organización conforme a su política de prevención y a su organización del trabajo en general. La elección entre las distintas modalidades organizativas que aparecen reguladas en la normativa preventiva (asunción por el propio empresario, trabajador designado, servicio de prevención propio o mancomunado, servicio de prevención ajeno) se mueve entre dos criterios: los riesgos existentes y la peligrosidad de las actividades que se desarrollen.

Es importante señalar que, aunque la empresa recurra a un servicio de prevención ajeno, es muy recomendable la existencia de un coordinador de prevención que sirva como interlocutor y apoyo logístico interno. También se establece la necesidad de contar con representantes de los trabajadores.

Ante lo anterior, se determina la concordancia de los tratados expuestos por los autores sobre la conceptualización de la organización, entendida esta, a criterio de la investigadora como la parte de la administración en donde se definen la estructura organizacional de la empresa estableciendo los criterios estratégicos para que la información fluya de una manera acorde y definida dentro de las gerencias de servicio lacustre de la industria petrolera división occidente.

\section{Comité de seguridad industrial}

Expone Cortés (2007) que el comité de seguridad y salud laboral debe participar en la elaboración, aprobación, puesta en marcha y evaluación de los mecanismos aplicados en el monitoreo y vigilancia epidemiológica de los procesos peligrosos de la empresa o centro de trabajo. Los grupos conformados hacen inspecciones a las instalaciones, evalúan las sugerencias de salud y seguridad ocupacional, analizan las causas de los accidentes y realizan recomendaciones. La conformación de dichas comisiones o comités de seguridad e higiene industrial en las condiciones expuestas, hacen su trabajo en la mayoría de los casos altamente fructífera.

Asimismo, afirma Marín (2006) que representan un ente paritario encargado de vigilar las condiciones y medio ambiente de trabajo, asistir y asesorar al empleador y a los trabajadores en la ejecución del programa de higiene y seguridad industrial. Para Muñoz (2008) la creación de comités de seguridad en las empresas es importante porque incorpora al trabajador a los programas de seguridad industrial de la empresa lo que permite intercambiar conocimientos y experiencias para lograr soluciones que contribuyan a mejorar las condiciones de seguridad.

A criterio de la investigadora, en las organizaciones el comité de seguridad y salud laboral elabora, aprueba, ejecuta y vigila todos los procesos, además favorece la incorporación de los trabajadores en los programas de seguridad industrial en las gerencias de servicio lacustre de la industria petrolera división occidente. 


\section{Responsabilidad}

Los autores Robbins y Ocultar y (2005), definen la responsabilidad como la obligación de desempeñar cualquier tarea asignada. Para Creus (2006), la responsabilidad viene dada según la definición organizativa de la empresa a la cual se le exige de todos sus colaboradores la máxima eficiencia en la ejecución de sus funciones.

Según Koontz y otros (2008), la responsabilidad es el conjunto de funciones definidas las cuales son asignadas a un individuo según el cargo o departamento al cual este pertenezca. Se hace evidente la concordancia entre los apartados expuestos por los autores sin embargo es importante acotar que Creus y Koontz hablan de definición de funciones mientras que Ocultar habla en términos de obligación.

Desde la perspectiva de la seguridad industrial e higiene ocupacional, la norma COVENIN 4001 (2000), establece que debe existir un representante el cual tenga conocimiento de las actividades de la organización y de los temas de salud y seguridad, para desarrollar de forma efectiva su cargo. Esta persona deberá asumir la responsabilidad de la implantación de las normas y la coordinación de las actividades de gestión preventiva de todos los servicios y grupos.

Pueden, no obstante, concurrir circunstancias en las que sus sustitutos designados actúen en su nombre sin que esto signifique que quede exento de su responsabilidad. Si el representante tiene que desempeñar otras funciones no se deberá producir ningún conflicto de intereses.

Según Janana (2012) considera, en adición a sus responsabilidades de seguridad, las organizaciones deben asegurarse de que no habrá condiciones que puedan dañar la salud de los trabajadores; de ahí que la primera obligación de un país sea mantener sus industrias en condiciones saludables y seguras.

A juicio de la investigadora, es sumamente importante para el sector de estudio que quienes gerencian la seguridad $y$ salud ocupacional conozcan las funciones que deben ejecutar en beneficio del establecimiento de condiciones seguras para todas las partes interesadas. Finalmente define responsabilidad como el grado de desempeño que posee un departamento de seguridad industrial e higiene ocupacional sobre las funciones que le fueron asignadas por las gerencias de servicio lacustre de la industria petrolera división occidente.

\section{Comunicación}

Para Robbins y Ocultar (2005), denominan la comunicación como la transferencia y la comprensión de significados. Si no se ha transmitido la información o ideas, la comunicación no se ha llevado a cabo. Su importancia radica en la compresión de los significados. Existen dos tipos de comunicación la comunicación interpersonal (la comunicación entre dos o más personas) y la comunicación organizacional (todos los patrones, redes y sistemas de comunicación de una organización).

Para Chiavenato (2009), la comunicación es la transferencia de información o de significado de una persona a otra. Dicho de otra forma, es el proceso por el cual se transmite información y significados de una persona a otra. Las organizaciones no pueden existir ni operar sin comunicación; está es la red que integra y coordina todas sus partes.

Según Koontz y otros (2008), la comunicación se define como la transferencia 
de información de un emisor a un receptor, el cual debe en condiciones de comprenderla. El proceso de comunicación dentro del sector bajo estudio es de suma importancia para la realización de las actividades cotidianas y las acciones preventivas que deben ser aplicadas.

En el contexto de la seguridad industrial e higiene ocupacional, debe existir una comunicación bidireccional efectiva, tal como lo establece la norma COVENIN 4001 (2000). Asimismo, la Ley de Prevención de Riesgos Laborales (1995), refiere que los trabajadores han de ser informados directamente por el empresario sobre los riesgos específicos de su puesto de trabajo, y de las medidas adoptadas y a adoptar.

De otro lado, podrán ser informados directamente por el empresario o a través de sus representantes sobre los riesgos generales de la empresa y sus medidas de prevención y protección, así como sobre las medidas de emergencia y en caso de riesgo grave e inminente. La información ha de ser bidireccional, también los trabajadores tienen el deber de informar de inmediato a su superior jerárquico y a los trabajadores designados para realizar actividades de protección y prevención o, en su caso, al servicio de prevención, acerca de cualquier situación que, a su juicio, entrañe un riesgo para la seguridad y salud de los trabajadores.

Cabe señalar que la información sobre la política preventiva de la empresa, y en general sobre todos los temas que atañen a las condiciones de trabajo del personal, debe ser realizada de forma transparente, evitando posibles tergiversaciones, para llegar a todos de la manera más directa posible.

Ante lo expuesto por los autores citados se establece concordancia en la esencia de la definición de la comunicación. Dicho esto, se conceptualiza la comunicación como el proceso de transferencia de información de forma clara y entendible entre un emisor y un receptor.

\section{MÉTODO}

En el trabajo que se presenta, se realiza un análisis del proceso de organización de la gestión de seguridad industrial e higiene ocupacional en la industria petrolera. Partiendo de este propósito, metodológicamente se enmarcó en una investigación de tipo descriptiva, con diseño no experimental, de campo y transeccional.

El universo de la investigación estuvo conformado por quince (15) gerentes y quince (15) supervisores de SIAHO, adscritos a las gerencias de Servicios Lacustre de la división occidente, específicamente en las Costas Occidental (Muelles Ali Primera, San Francisco, Sucre y Libertador) y Oriental del Lago (Muelles la Salina, Tía Juana, Simón Bolívar, Luisa Cáceres de Arismendi, Soberanía, Patria Grande, Rafael Urdaneta, Ciudad Ojeda, Lagunillas Norte, Lagunillas Sur y Bachaquero I).

Para la recolección de los datos, y consecuente con lo anterior, la técnica empleada fue la encuesta. Asimismo, en concordancia con la técnica seleccionada, el instrumento empleado fue el cuestionario, el cual constó de nueve ítems, utilizando una escala dicotómica. En este caso, para la validez de contenido, se sometió a la técnica del juicio de expertos. Una vez consideradas las observaciones de los expertos que estuvieron centradas en redacción (estilo y forma de acuerdo a la escala utilizada) se procedió a realizar una prueba piloto para determinar la confiabilidad, aplicándose el método del Coeficiente Küder - Richardson, el cual arrojó un 
coeficiente de 0,85 significando una alta confiabilidad.

Luego que la información fue tabulada, ordenada y sometida a tratamiento por técnicas de análisis matemático de carácter estadístico, se tabularon y analizaron los datos asociados al cuestionario. El procesamiento de los datos se realizó a través de la estadística descriptiva, utilizando las frecuencias relativas por medio de la aplicación del baremo de interpretación correspondiente, mostrado en el cuadro 1.

Cuadro 1. Baremo de interpretación

\begin{tabular}{ccc}
\hline Alternativa de respuesta & Intervalo & Categoría \\
\hline $\mathrm{Si}$ & $75 \% \leq X<100 \%$ & Alta presencia \\
$\mathrm{Si}$ & $50 \% \leq X<75 \%$ & Moderada presencia \\
$\mathrm{Si}$ & $25 \% \leq X<50 \%$ & Baja presencia \\
$\mathrm{Si}$ & $0 \% \leq X<25 \%$ & Muy Baja presencia \\
\hline
\end{tabular}

Fuente: La investigadora (2019)

RESULTADOS mecanismos para el monitoreo de los riesgos de

En referencia a los resultados que permiten conocer ¿cómo es el proceso de organización en la gestión de seguridad industrial e higiene ocupacional presente en las gerencias de servicio lacustre de la industria petrolera división occidente?, los datos de la tabla 1 evidencian una moderada presencia (60\%) del indicador comité de seguridad industrial, opinando los encuestados en un $63 \%$ que si cuenta la empresa con un comité de seguridad y salud laboral, por otra parte el $53 \%$ dicen que participa el comité de seguridad en establecer la empresa, asimismo el 63\% afirma que el comité hace inspecciones a las instalaciones de la empresa.

A criterio de la investigadora, esto representa para las gerencias de servicio lacustre observadas, una oportunidad para establecer estrategias en función de lograr grupos conformados para hacer inspecciones a las instalaciones, evaluar las sugerencias de salud y seguridad ocupacional, así como analizar las causas de los accidentes a fin de realizar recomendaciones efectivas.

Tabla 1: Indicador: Comité de seguridad industrial

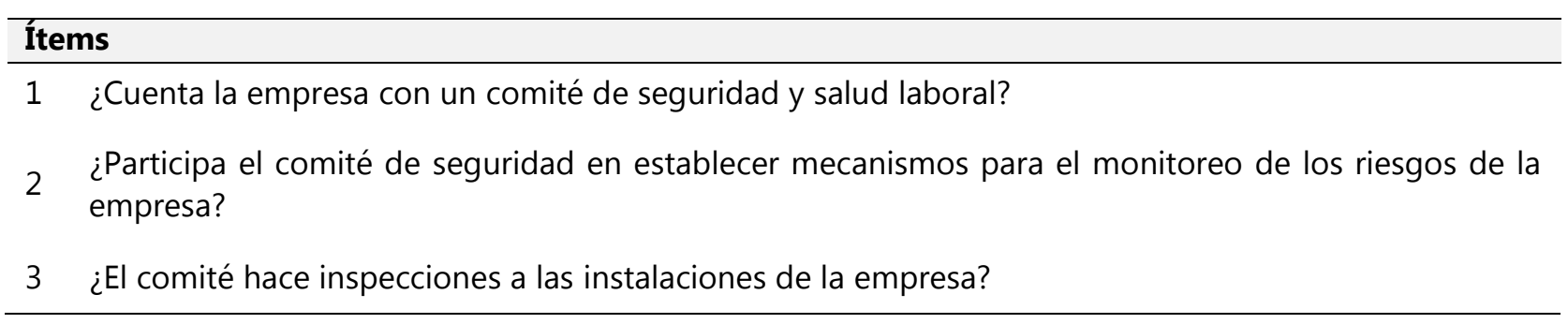




\begin{tabular}{|c|c|c|c|c|c|c|}
\hline \multirow{3}{*}{ Alternativa } & \multicolumn{6}{|c|}{ Ítems } \\
\hline & \multicolumn{2}{|c|}{1} & \multicolumn{2}{|c|}{2} & \multicolumn{2}{|c|}{3} \\
\hline & $\mathbf{F a}$ & Fr & $\mathbf{F a}$ & Fr & $\mathrm{Fa}$ & Fr \\
\hline $\mathrm{Si}$ & 19 & $63 \%$ & 16 & $53 \%$ & 19 & $63 \%$ \\
\hline NO & 11 & $37 \%$ & 14 & $47 \%$ & 11 & $37 \%$ \\
\hline Total & 30 & $100 \%$ & 30 & $100 \%$ & 30 & $100 \%$ \\
\hline Categoría & \multirow{3}{*}{\multicolumn{2}{|c|}{ Moderada presencia }} & \multirow{3}{*}{\multicolumn{2}{|c|}{$\begin{array}{c}\text { Moderada presencia } \\
60 \% \\
\text { Moderada presencia }\end{array}$}} & \multirow{3}{*}{\multicolumn{2}{|c|}{ Moderada presencia }} \\
\hline Promedio & & & & & & \\
\hline Categoría & & & & & & \\
\hline
\end{tabular}

Fuente: Elaboración propia (2019)

Los resultados antes descritos, se corresponden parcialmente con los postulados de Marín (2006) para quien el comité de seguridad industrial representa un ente paritario encargado de vigilar las condiciones y medio ambiente de trabajo, asistir y asesorar al empleador y a los trabajadores en la ejecución del programa de higiene y seguridad industrial.

De igual manera, alcanzan a validar lo establecido por la investigadora, cuando afirma que el comité de seguridad y salud laboral debe elaborar, aprobar, ejecutar y vigilar todos los procesos, además de favorecer la incorporación de los trabajadores en los programas de seguridad industrial en las gerencias de servicio lacustre de la industria petrolera división occidente.
Los valores promedio mostrados en la tabla 2, revelan que el $73 \%$ de los encuestados consideran la responsabilidad como indicador del proceso de organización en la gestión de seguridad industrial e higiene ocupacional en las gerencias analizadas, se realiza con moderada presencia. Se muestra para este indicador con alta presencia, al considerar en un $80 \%$ que los integrantes de la gestión SIHO tienen definidas las funciones del departamento, no obstante, con moderada presencia el $73 \%$ opinan que los integrantes de la gestión SIHO tienen definido el cargo que ejerce y un $67 \%$ afirman que las responsabilidades se encuentran distribuidas equitativamente entre los integrantes del departamento de SIHO de la empresa. 
Tabla 2: Indicador: Responsabilidad

\begin{tabular}{|c|c|c|c|c|c|c|}
\hline \multicolumn{7}{|l|}{ Ítems } \\
\hline \multicolumn{7}{|c|}{$\begin{array}{l}\text { ¿Los integrantes de la gestión SIHO tienen definidas las funciones del departamento? } \\
\text { ¿Los integrantes de la gestión SIHO tienen definido el cargo que ejerce? } \\
\text { ¿Las responsabilidades se encuentran distribuidas equitativamente entre los integrantes del } \\
\text { departamento de SIHO de la empresa? }\end{array}$} \\
\hline \multirow{3}{*}{ Alternativa } & \multicolumn{6}{|c|}{ Ítems } \\
\hline & \multicolumn{2}{|c|}{4} & \multicolumn{2}{|c|}{5} & \multicolumn{2}{|c|}{6} \\
\hline & $\mathbf{F a}$ & $\mathbf{F r}$ & $\mathbf{F a}$ & $\mathbf{F r}$ & $\mathbf{F a}$ & Fr \\
\hline $\mathrm{Si}$ & 24 & $80 \%$ & 22 & $73 \%$ & 20 & $67 \%$ \\
\hline NO & 6 & $20 \%$ & 8 & $27 \%$ & 10 & $33 \%$ \\
\hline Total & 30 & $100 \%$ & 30 & $100 \%$ & 30 & $100 \%$ \\
\hline Categoría & \multicolumn{2}{|c|}{ Alta presencia } & \multicolumn{2}{|c|}{ Moderada presencia } & \multirow{2}{*}{\multicolumn{2}{|c|}{ Moderada presencia }} \\
\hline Promedio & \multicolumn{4}{|c|}{$73 \%$} & & \\
\hline Categoría & \multicolumn{6}{|c|}{ Moderada presencia } \\
\hline
\end{tabular}

Fuente: Elaboración propia (2019)

A tal efecto, según la investigadora quienes integran las gerencias de servicio lacustre de la industria petrolera división occidente, deben aplicar un conjunto de funciones definidas las cuales corresponden a cada individuo según el cargo o departamento al cual este pertenezca.

De manera que, según estos resultados, existe parcial congruencia entre lo evidenciado y lo expuesto por la norma COVENIN 4001 (2000), donde se establece que debe existir un representante el cual tenga conocimiento de las actividades de la organización y de los temas de salud y seguridad, para desarrollar de forma efectiva su cargo.

Al mismo tiempo, coinciden con el juicio de la investigadora, cuando considera que es importante para el sector de estudio que quienes gerencian la seguridad $y$ salud ocupacional conozcan las funciones que deben ejecutar en beneficio del establecimiento de condiciones seguras para todas las partes interesadas. Aunado a ello la investigadora, define la responsabilidad como el grado de desempeño que posee un departamento de seguridad industrial e higiene ocupacional sobre las funciones que le fueron asignadas por las gerencias de servicio lacustre de la industria petrolera división occidente.

En el mismo orden de ideas, se visualiza en la tabla 3 para el indicador comunicación, un promedio de $72 \%$ ubicándolo en la categoría de moderada presencia. Esta situación es explicada por el comportamiento de las respuestas dadas a cada ítem, en las cuales se observa alta presencia al considerar el $87 \%$ de los encuestados que la gestión SIHO posee definidos los procedimientos de comunicación que aseguran la transmisión de información de forma adecuada entre todas las partes, no obstante con moderada presencia el $63 \%$ afirma que la comunicación entre los integrantes del departamento de SIHO es oportuna y un $67 \%$ asegura que la gestión SIHO informa oportunamente a los directivos sobre cambios necesarios de los procesos en materia de seguridad industrial e higiene ocupacional. 
Tabla 3: Indicador: Comunicación

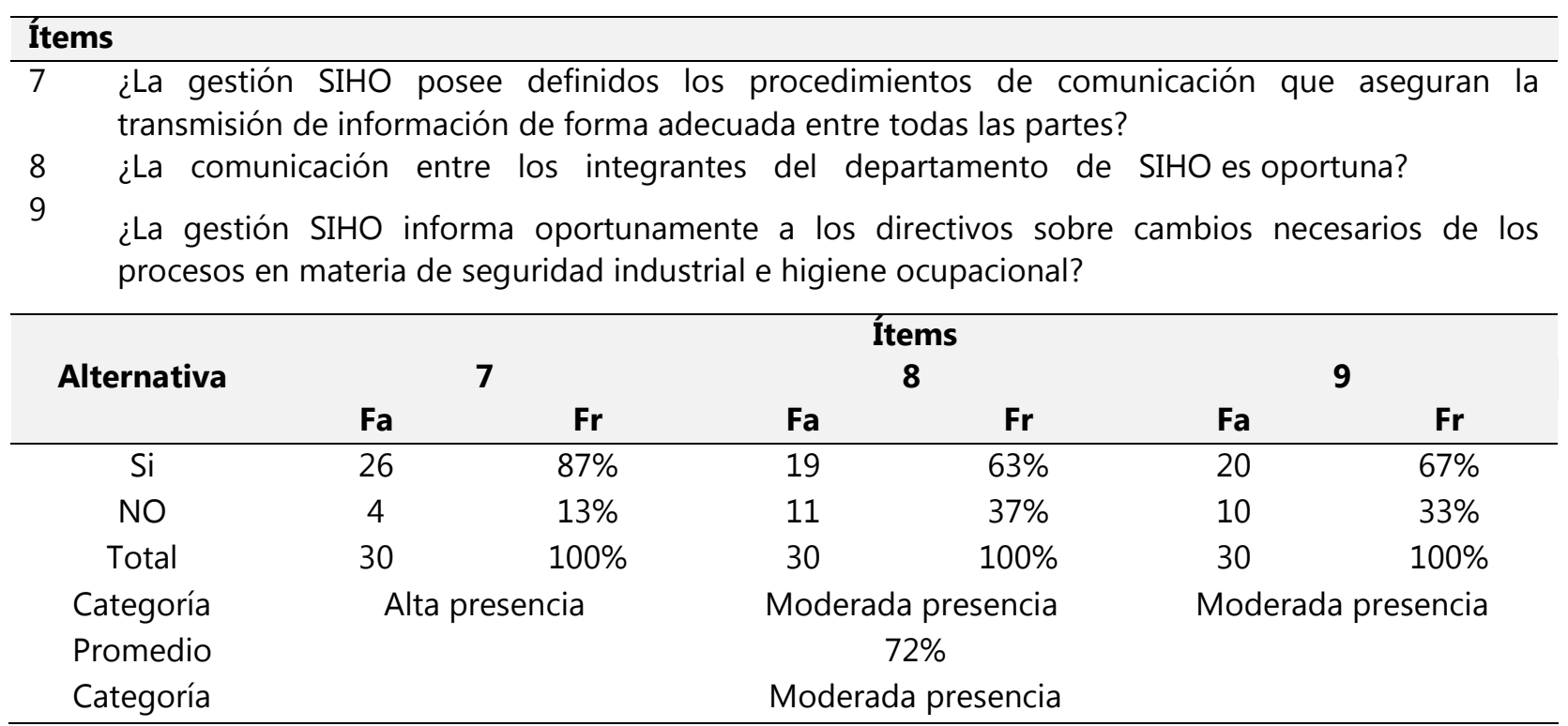

Fuente: Elaboración propia (2019)

A juicio de la investigadora es una oportunidad de mejora en las gerencias analizadas, por cuanto se evidencia que en el servicio lacustre de la industria petrolera división occidente moderadamente se transmite información, elemento importante para que las organizaciones realicen sus actividades cotidianas y las acciones preventivas que deben ser aplicadas.

Las afirmaciones precedentes alcanzan a validar los postulados de la Ley de Prevención de Riesgos Laborales (1995), la cual refiere que los trabajadores han de ser informados directamente por el empresario sobre los riesgos específicos de su puesto de trabajo, y de las medidas adoptadas y a adoptar.

De manera similar, validan a la investigadora, para quien, en el sector bajo estudio, la información sobre la política preventiva de la empresa, y en general sobre todos los temas que atañen a las condiciones de trabajo del personal, debe ser realizada de forma transparente, evitando posibles tergiversaciones, para llegar a todos de la manera más directa posible.

Como se puede apreciar en la tabla 4, se presenta el resumen para la dimensión proceso de organización, la cual muestra un valor de $68 \%$ de respuestas afirmativas, reflejando, de acuerdo a la opinión de los encuestados, moderada presencia del proceso de organización en la gestión de seguridad industrial e higiene ocupacional en las gerencias de servicio lacustre de la industria petrolera división occidente. 
Tabla 4: Dimensión: Proceso de organización

\begin{tabular}{lllll}
\hline Indicador & Promedio & Categoría & Promedio & Categoría \\
\hline Comité de seguridad industrial & $60 \%$ & Moderada presencia & & \\
Responsabilidad & $73 \%$ & Moderada presencia & $\mathbf{6 8 \%}$ & $\begin{array}{l}\text { Moderada } \\
\text { presencia }\end{array}$ \\
Comunicación & $72 \%$ & Moderada presencia & & \\
\hline
\end{tabular}

Fuente: Elaboración propia (2019)

Al ver los resultados se establecen con moderada presencia los indicadores comité de seguridad industrial, responsabilidad $y$ comunicación como parte del proceso de organización en la gestión de seguridad industrial e higiene ocupacional en las gerencias de servicio lacustre de la industria petrolera división occidente. A juicio de la investigadora estos aspectos deben ser considerados como oportunidades de mejora por cuanto toda empresa debe determinar y definir la manera en que, desde el punto de vista organizacional, funcionará distribuyendo las tareas y los puestos de trabajo para cada uno de los eslabones que la deben conformar.

Estos señalamientos validan, medianamente, lo planteado por Hernández y otros (2003) quienes refieren que se debe disponer de una organización y de los medios necesarios que aseguren el cumplimiento del deber de protección empresarial (conjunto de relaciones y personas que integradas en la estructura organizativa de la empresa asegura el cumplimiento de los objetivos preventivos).

En este orden a juicio de la investigadora, la organización forma parte de la administración, en donde se define la estructura organizacional de la empresa estableciendo los criterios estratégicos para que la información fluya de una manera acorde y definida dentro de las gerencias de servicio lacustre de la industria petrolera división occidente.

\section{CONCLUSIONES}

En lo que concierne al objetivo, donde se analizó el proceso de organización de la gestión de seguridad industrial e higiene ocupacional en la industria petrolera, se concluye que estas gerencias otorgan moderada presencia al comité de seguridad industrial, responsabilidad y comunicación como indicadores del proceso.

Al detalle, se identificó que en las gerencias de servicio lacustre observadas el comité de seguridad industrial representa una oportunidad de mejora para establecer estrategias en función de lograr grupos conformados para hacer inspecciones a las instalaciones, evaluar las sugerencias de salud y seguridad ocupacional, así como analizar las causas de los accidentes a fin de realizar recomendaciones efectivas.

Respecto a la responsabilidad se concluye que quienes integran las gerencias de servicio lacustre de la industria petrolera división occidente, deben aplicar un conjunto de funciones definidas las cuales corresponden a cada individuo según el cargo o departamento al cual este pertenezca. Asimismo, los resultados revelaron en cuanto al indicador comunicación, que este representa una oportunidad de mejora en las gerencias analizadas, por cuanto se evidencia que en el servicio lacustre de la industria petrolera división occidente moderadamente se transmite información, elemento importante para que las organizaciones realicen sus actividades 
cotidianas y las acciones preventivas que deben ser aplicadas.

\section{REFERENCIAS}

Aguilar, E. (2011). Desarrollo de un modelo de gestión de seguridad e higiene industrial. Primera edición. Editorial EAE. España

Chiavenato, I. (2009) Introducción a la Teoría Administrativa. Editorial Mc Graw Hill. México

Cortés, J. (2007). Seguridad e Higiene del Trabajo. Editorial Tébar, Novena edición. Madrid.

Creus, A. (2006). Gestión de la Prevención. Ediciones CEAC, Primera edición. Barcelona

Hernández, A.; Malfavón, N.; y Fernández, G. (2003). Seguridad e higiene industrial. Editorial Limusa. México

Hernández, A; Zúñiga, N; Malfavon, G. (2005). Seguridad e Higiene Industrial. 6ta Edición. Editorial Limusa. México

Janania, A. (2012). Manual de Seguridad e Higiene Industrial, Limusa. México D.F.

Koontz, H., Weihrich, $H$. y Cannice, M. (2008). Administración: una Perspectiva Global. McGraw Hill, Decimotercera edición. México D.F

Ley de Prevención de Riesgos Laborales. (1995). Ley 31/1995, de 8 de noviembre, de Prevención de Riesgos Laborales. B.O.E. núm. 269, de 10 de noviembre de 1995. Disponible en: http://www.prevenciondocente.com/leyprl.p $\mathrm{df}$

Marín, A. (2006). Seguridad industrial. Manual para a formación de ingenieros. Universidad Rey Juan Carlos. Editorial Oylinson S.L. Madrid. España

Norma Venezolana COVENIN 4001 (2000). Sistema de Gestión de Seguridad e Higiene Ocupacional. Requisitos: Disponible en;

http://www.cpzulia.org/ARCHIVOS_SSA/4001 2000_SGSHO.pdf

Norma Venezolana COVENIN 2260 (2004). Sistema de Gestión de Seguridad e Higiene Ocupacional. Requisitos: Disponible en:

http://www.sencamer.gob.ve/sencamer/nor mas/2260-04.pdf

PDVSA página oficial de internet (2019). Disponible en: http://www.pdvsa.com/index.php?tpl=interfa ce.sp/design/readmenuprinc_eps.tpl.html\&n ewsid_temas $=220$

Peralta U. (2010). Seguridad e higiene industrial. Editorial Mc Graw Hill. México

Robbins S. y Ocultar M (2005). Administración, Atlacomulco, Pearson Educación, Octava edición. México 